\title{
ON CONSTRUCTIVE CONNECTIVES AND SYSTEMS
}

\author{
ARNON AVRON AND ORI LAHAV
}

School of Computer Science, Tel Aviv University, Israel

e-mail address: \{aa,orilahav\}@post.tau.ac.il

\begin{abstract}
Canonical inference rules and canonical systems are defined in the framework of non-strict single-conclusion sequent systems, in which the succeedents of sequents can be empty. Important properties of this framework are investigated, and a general nondeterministic Kripke-style semantics is provided. This general semantics is then used to provide a constructive (and very natural), sufficient and necessary coherence criterion for the validity of the strong cut-elimination theorem in such a system. These results suggest new syntactic and semantic characterizations of basic constructive connectives.
\end{abstract}

\section{INTRODUCTION}

There are two traditions concerning the definition and characterization of logical connectives. The better known one is the semantic tradition, which is based on the idea that an $n$-ary connective $\diamond$ is defined by the conditions which make a sentence of the form $\diamond\left(\varphi_{1}, \ldots, \varphi_{n}\right)$ true. The other is the proof-theoretic tradition (originated from [9] - see e.g. 14 for discussions and references). This tradition implicitly divides the connectives into basic connectives and compound connectives, where the latter are defined in terms of the basic ones. The meaning of a basic connective, in turn, is determined by a set of derivation rules which are associated with it. Here one usually has in mind a natural deduction or a sequent system, in which every logical rule is an introduction rule (or perhaps an elimination rule, in the case of natural deduction) of some unique connective. However, it is well-known that not every set of rules can be taken as a definition of a basic connective. A minimal requirement is that whenever some sentence involving exactly one basic connective is provable, then it has a proof which involves no other connectives. In "normal" sequent systems, in which every rule except cut has the subformula property, this condition is guaranteed by a cut-elimination theorem. Therefore only sequent systems for which such a theorem obtains are considered as useful for defining connectives.

In [3] the semantic and the proof-theoretic traditions were shown to be equivalent for a a large family of what may be called semi-classical connectives (which includes all the classical connectives, as well as many others). In these papers multiple-conclusion canonical (= 'ideal') propositional rules and systems were defined in precise terms. A simple coherence

1998 ACM Subject Classification: F.4.1, I.2.3.

Key words and phrases: sequent calculus, cut-elimination, nonclassical logics, non-deterministic semantics, Kripke semantics. 
criterion for the non-triviality of such a system was given, and it was shown that a canonical system is coherent if and only if it admits cut-elimination. Semi-classical connectives were characterized using canonical rules in coherent canonical systems. In addition, each of these connectives was given a semantic characterization. This characterization uses twovalued non-deterministic truth-tables - a natural generalization of the classical truth-tables. Moreover, it was shown there how to translate a semantic definition of a connective to a corresponding proof-theoretic one, and vice-versa 1

In this paper we attempt to provide similar characterizations for the class of basic constructive connectives.

What exactly is a constructive connective? Several different answers to this question have been given in the literature, each adopting either of the traditions described above (but not both!). Thus in [12] McCullough gave a purely semantic characterization of constructive connectives, using a generalization of the Kripke-style semantics for intuitionistic logic. On the other hand Bowen suggested in [7] a quite natural proof-theoretic criterion for (basic) constructivity: an $n$-ary connective $\diamond$, defined by a set of sequent rules, is constructive if whenever a sequent of the form $\Rightarrow \diamond\left(\varphi_{1}, \ldots, \varphi_{n}\right)$ is provable, then it has a proof ending by an application of one of right-introduction rules for $\diamond$.

In what follows we generalize and unify the syntactic and the semantic approaches by adapting the ideas and methods used in [3]. The crucial observation on which our theory is based is that every connective of a "normal" single-conclusion sequent system that admits cut-elimination is necessarily constructive according to Bowen's criterion (because without using cuts, the only way to derive $\Rightarrow \diamond\left(\varphi_{1}, \ldots, \varphi_{n}\right)$ in such a system is to prove first the premises of one of its right-introduction rules). This indicates that only single-conclusion sequent rules are useful for defining constructive connectives. In addition, for defining basic connectives, only canonical derivation rules (in a sense similar to that used in [3]) should be used. Therefore, our proof-theoretic characterization of basic constructive connectives is done using cut-free single-conclusion canonical systems. These systems are the natural constructive counterparts of the multiple-conclusion canonical systems of [3]. On the other hand, McCullough's work suggests that an appropriate counterpart of the semantics of nondeterministic truth-tables should be given by a non-deterministic generalization of Kripkestyle semantics.

General single-conclusion canonical rules and systems were first introduced and investigated in [4. A general non-deterministic Kripke-style semantics for such systems was also developed there, and a constructive necessary and sufficient coherence criterion for their non-triviality was provided. Moreover: it was shown that a system of this kind admits a strong form of cut-elimination iff it is coherent. However, 44 dealt only with strict single-conclusion systems, in which the succeedents of sequents contain exactly one formula. Unfortunately, in such a framework it is impossible to have canonical rules even for a crucial connective like intuitionistic negation. To solve this, we move here to Gentzen's original (non-strict) single-conclusion framework, in which the succeedents of sequents contain at most one formula. There is a price to pay, though, for this extension of the framework. As we show below, in this more general framework we lose the equivalence between the simple coherence criterion of [3, 4] and non-triviality, as well as the equivalence proved there between simple cut-elimination and strong cut-elimination. Hence the theory needs some major changes.

\footnotetext{
${ }^{1}$ It might be interesting to note that every connective in this framework can be viewed as basic.
} 
In the rest of this paper we first redefine the notions of a canonical inference rule and a canonical system in the framework of non-strict single-conclusion sequent systems. Then we turn to the semantic point of view, and present a corresponding general nondeterministic Kripke-style semantics. We show that every canonical system induces a class of non-deterministic Kripke-style frames, for which it is strongly sound and complete. This general semantics is then used to show that a canonical system $\mathbf{G}$ is coherent iff it admits a strong form of non-triviality, and this happens iff the strong cut-elimination theorem is valid for $\mathbf{G}$.

Taken together, the results of this paper suggest that a basic constructive connective is a connective that can be defined using a set of canonical rules in a coherent (non-strict) single-conclusion sequent system. We show that this class is broader than that suggested in [12], and includes connectives that cannot be expressed by the four basic intuitionistic connectives. Examples include the "converse non-implication" and "not-both" connectives from [7], as well as the weak implication of primal intuitionistic logic from [10]. These connectives were left out by McCullough's deterministic semantic characterization because their semantics is strictly non-deterministic.

\section{Preliminaries}

In what follows $\mathcal{L}$ is a propositional language, $\mathcal{F}$ is its set of wffs, $p, q$ denote atomic formulas, $\psi, \varphi, \theta$ denote arbitrary formulas (of $\mathcal{L}$ ), $\mathcal{T}$ and $\mathcal{U}$ denote subsets of $\mathcal{F}, \Gamma, \Delta, \Sigma, \Pi$ denote finite subsets of $\mathcal{F}$, and $E, F$ denote subsets of $\mathcal{F}$ with at most one element. We assume that the atomic formulas of $\mathcal{L}$ are $p_{1}, p_{2}, \ldots$ (in particular: $p_{1}, \ldots, p_{n}$ are the first $n$ atomic formulas of $\mathcal{L})$.

Notation. For convenience we sometimes discard parentheses for sets, and write e.g. just $\psi$ instead of $\{\psi\}$. We also employ other standard abbreviations, like $\Gamma, \Delta$ instead of $\Gamma \cup \Delta$.

Definition 2.1. A Tarskian consequence relation (tcr for short) for $\mathcal{L}$ is a binary relation $\vdash$ between sets of formulas of $\mathcal{L}$ and formulas of $\mathcal{L}$ that satisfies the following conditions:

Strong Reflexivity: $\quad$ if $\varphi \in \mathcal{T}$ then $\mathcal{T} \vdash \varphi$.

Monotonicity: $\quad$ if $\mathcal{T} \vdash \varphi$ and $\mathcal{T} \subseteq \mathcal{T}^{\prime}$ then $\mathcal{T}^{\prime} \vdash \varphi$.

Transitivity (cut): $\quad$ if $\mathcal{T} \vdash \psi$ and $\mathcal{T}, \psi \vdash \varphi$ then $\mathcal{T} \vdash \varphi$.

In the non-strict framework, it is natural to extend Definition 2.1 as follows:

Definition 2.2. An Extended Tarskian consequence relation (etcr for short) for $\mathcal{L}$ is a binary relation $\vdash$ between sets of formulas of $\mathcal{L}$ and singletons or empty sets of formulas of $\mathcal{L}$ that satisfies the following conditions:

Strong Reflexivity: $\quad$ if $\varphi \in \mathcal{T}$ then $\mathcal{T} \vdash \varphi$.

Monotonicity: $\quad$ if $\mathcal{T} \vdash E, \mathcal{T} \subseteq \mathcal{T}^{\prime}$, and $E \subseteq E^{\prime}$, then $\mathcal{T}^{\prime} \vdash E^{\prime}$

Transitivity (cut): $\quad$ if $\mathcal{T} \vdash \psi$ and $\mathcal{T}, \psi \vdash E$ then $\mathcal{T} \vdash E$.

Intuitively, " $\mathcal{T} \vdash$ " means that $\mathcal{T}$ is inconsistent (i.e. $\mathcal{T} \vdash \varphi$ for every formula $\varphi$ ).

Definition 2.3. An $\mathcal{L}$-substitution is a function $\sigma: \mathcal{F} \rightarrow \mathcal{F}$, such that for every $n$-ary connective $\diamond$ of $\mathcal{L}$, we have: $\sigma\left(\diamond\left(\psi_{1}, \ldots, \psi_{n}\right)\right)=\diamond\left(\sigma\left(\psi_{1}\right), \ldots, \sigma\left(\psi_{n}\right)\right)$. Obviously, a substitution is determined by the values it assigns to atomic formulas. A substitution is extended to sets of formulas in the obvious way: $\sigma(\mathcal{T})=\{\sigma(\varphi) \mid \varphi \in \mathcal{T}\}$ (in particular, $\sigma(\emptyset)=\emptyset$ ). 
Definition 2.4. An etcr $\vdash$ for $\mathcal{L}$ is structural if for every $\mathcal{L}$-substitution $\sigma$ and every $\mathcal{T}$ and $E$, if $\mathcal{T} \vdash E$ then $\sigma(\mathcal{T}) \vdash \sigma(E)$. $\vdash$ is finitary iff the following condition holds for every $\mathcal{T}$ and $E$ : if $\mathcal{T} \vdash E$ then there exists a finite $\Gamma \subseteq \mathcal{T}$ such that $\Gamma \vdash E$. $\vdash$ is consistent (or non-trivial) if $p_{1} \nvdash p_{2}$.

It is easy to see that there are exactly four inconsistent structural etcrs in any given language: $\mathcal{T} \vdash E$ for every $\mathcal{T}$ and $E ; \mathcal{T} \vdash E$ for every $E$ and nonempty $\mathcal{T} ; \mathcal{T} \vdash E$ for every $\mathcal{T}$ and nonempty $E$; and $\mathcal{T} \vdash E$ for every nonempty $\mathcal{T}$ and nonempty $E$. These etcrs are obviously trivial, so we exclude them from our definition of an extended logic:

Definition 2.5. A propositional extended logic is a pair $\langle\mathcal{L}, \vdash\rangle$, where $\mathcal{L}$ is a propositional language, and $\vdash$ is an etcr for $\mathcal{L}$ which is structural, finitary, and consistent.

Sequents, which are the main tool for introducing extended logics, are defined as follows:

Definition 2.6. A non-strict single-conclusion sequent is an expression of the form $(\Gamma \Rightarrow E)$ where $\Gamma$ and $E$ are finite sets of formulas, and $E$ is either a singleton or empty. A nonstrict single-conclusion Horn clause is a non-strict single-conclusion sequent which consists of atomic formulas only.

Convention. From now on, by "sequent (clause)" we shall mean "non-strict single-conclusion sequent (Horn clause)".

Definition 2.7. A sequent of the form $(\Gamma \Rightarrow\{\varphi\})$ is called definite. A sequent of the form $(\Gamma \Rightarrow \emptyset)$ is called negative.

Notation. We mainly use $s$ to denote a sequent and $\mathcal{S}$ to denote a set of sequents. We usually omit the outermost parentheses of sequents to improve readability. For convenience, we shall denote a sequent of the form $\Gamma \Rightarrow \emptyset$ by $\Gamma \Rightarrow$, and a sequent of the form $\Gamma \Rightarrow\{\varphi\}$ by $\Gamma \Rightarrow \varphi$.

\section{Canonical Systems}

The following definitions formulate in exact terms the structure of sequent rules (and systems) that can be used to define basic constructive connectives. We first define rightintroduction rules and their applications, and then deal with left-introduction rules.

\section{Definition 3.1.}

(1) A single-conclusion canonical right-introduction rule for a connective $\diamond$ of arity $n$ is an expression of the form:

$$
\left\{\Pi_{i} \Rightarrow E_{i}\right\}_{1 \leq i \leq m} / \Rightarrow \diamond\left(p_{1}, \ldots, p_{n}\right)
$$

where $m \geq 0$, and $\Pi_{i} \cup E_{i} \subseteq\left\{p_{1}, \ldots, p_{n}\right\}$ for every $1 \leq i \leq m$. The clauses $\Pi_{i} \Rightarrow E_{i}$ $(1 \leq i \leq m)$ are the premises of the rule, while $\Rightarrow \diamond\left(p_{1}, \ldots, p_{n}\right)$ is its conclusion.

(2) An application of the rule $\left\{\Pi_{i} \Rightarrow E_{i}\right\}_{1 \leq i \leq m} / \Rightarrow \diamond\left(p_{1}, \ldots, p_{n}\right)$ is any inference step of the form:

$$
\frac{\left\{\Gamma, \sigma\left(\Pi_{i}\right) \Rightarrow \sigma\left(E_{i}\right)\right\}_{1 \leq i \leq m}}{\Gamma \Rightarrow \sigma\left(\diamond\left(p_{1}, \ldots, p_{n}\right)\right)}
$$

where $\Gamma$ is a finite set of formulas and $\sigma$ is an $\mathcal{L}$ - substitution. 
A canonical right-introduction rule may have negative premises (negative sequents serving as premises). Obviously, in applications of such a rule, a right context formula cannot be added to its negative premises. Left-introduction rules are somewhat more complicated, since in their applications it is not impossible to add a right context formula to the negative premises and to the conclusion. However, in the general case there might also be negative premises which do not allow such an addition of a right context. Accordingly, in what follows we split the set of premises of a canonical left-introduction rule into two sets: hard premises which do not allow right context, and soft premises, which do allow it.

\section{Definition 3.2.}

(1) A single-conclusion canonical left-introduction rule for a connective $\diamond$ of arity $n$ is an expression of the form:

$$
\left\langle\left\{\Pi_{i} \Rightarrow E_{i}\right\}_{1 \leq i \leq m},\left\{\Sigma_{j} \Rightarrow\right\}_{1 \leq j \leq k}\right\rangle / \diamond\left(p_{1}, \ldots, p_{n}\right) \Rightarrow
$$

where $m, k \geq 0, \Pi_{i} \cup E_{i} \subseteq\left\{p_{1}, \ldots, p_{n}\right\}$ for $1 \leq i \leq m$, and $\Sigma_{j} \subseteq\left\{p_{1}, \ldots, p_{n}\right\}$ for $1 \leq j \leq k$. The clauses $\Pi_{i} \Rightarrow E_{i}(1 \leq i \leq m)$ are called the hard premises of the rule, $\Sigma_{j} \Rightarrow(1 \leq j \leq k)$ are its soft premises, and $\diamond\left(p_{1}, \ldots, p_{n}\right) \Rightarrow$ is its conclusion.

(2) An application of the rule $\left\langle\left\{\Pi_{i} \Rightarrow E_{i}\right\}_{1 \leq i \leq m},\left\{\Sigma_{i} \Rightarrow\right\}_{1 \leq i \leq k}\right\rangle / \diamond\left(p_{1}, \ldots, p_{n}\right) \Rightarrow$ is any inference step of the form:

$$
\frac{\left\{\Gamma, \sigma\left(\Pi_{i}\right) \Rightarrow \sigma\left(E_{i}\right)\right\}_{1 \leq i \leq m} \quad\left\{\Gamma, \sigma\left(\Sigma_{i}\right) \Rightarrow E\right\}_{1 \leq i \leq k}}{\Gamma, \sigma\left(\diamond\left(p_{1}, \ldots, p_{n}\right)\right) \Rightarrow E}
$$

where $\Gamma \Rightarrow E$ is an arbitrary sequent, and $\sigma$ is an $\mathcal{L}$ - substitution.

Remark 3.3. Note that definite premises of a canonical left-introduction rules are all hard premises, as they do not allow the addition of a right context.

Convention. From now on, by "canonical right-introduction (left-introduction) rule" we shall mean "single-conclusion canonical right-introduction (left-introduction) rule".

Examples 3.4. We give some examples for canonical rules.

Implication: The two usual rules for implication are:

$$
\left\langle\left\{\Rightarrow p_{1}\right\},\left\{p_{2} \Rightarrow\right\}\right\rangle / p_{1} \supset p_{2} \Rightarrow \quad \text { and } \quad\left\{p_{1} \Rightarrow p_{2}\right\} / \Rightarrow p_{1} \supset p_{2}
$$

Applications of these rules have the form:

$$
\frac{\Gamma \Rightarrow \psi \quad \Gamma, \varphi \Rightarrow E}{\Gamma, \psi \supset \varphi \Rightarrow E} \quad \frac{\Gamma, \psi \Rightarrow \varphi}{\Gamma \Rightarrow \psi \supset \varphi}
$$

Absurdity]: In intuitionistic logic there is no right-introduction rule for the absurdity constant $\perp$, and there is exactly one left-introduction rule for it:

$$
\langle\emptyset, \emptyset\rangle / \perp \Rightarrow
$$

Applications of this rule provide new axioms:

$$
\Gamma, \perp \Rightarrow E
$$

Negation: Unlike in [4], in this new framework it is possible to handle negation as a basic connective, using the following standard rules:

$$
\left\langle\left\{\Rightarrow p_{1}\right\}, \emptyset\right\rangle / \neg p_{1} \Rightarrow \text { and }\left\{p_{1} \Rightarrow\right\} / \Rightarrow \neg p_{1}
$$

Applications of these rules have the form:

$$
\frac{\Gamma \Rightarrow \psi}{\Gamma, \neg \psi \Rightarrow E} \quad \frac{\Gamma, \psi \Rightarrow}{\Gamma \Rightarrow \neg \psi}
$$


Semi-Implication: In [10] $\leadsto$ was introduced using the following two rules:

$$
\left\langle\left\{\Rightarrow p_{1}\right\},\left\{p_{2} \Rightarrow\right\}\right\rangle / p_{1} \leadsto p_{2} \Rightarrow \quad \text { and } \quad\left\{\Rightarrow p_{2}\right\} / \Rightarrow p_{1} \leadsto p_{2}
$$

Applications of these rules have the form:

$$
\frac{\Gamma \Rightarrow \psi \quad \Gamma, \varphi \Rightarrow E}{\Gamma, \psi \sim \varphi \Rightarrow E} \quad \frac{\Gamma \Rightarrow \varphi}{\Gamma \Rightarrow \psi \leadsto \varphi}
$$

Affirmation: Let the connective $\triangleright$ be defined using the following rules:

$$
\left\langle\emptyset,\left\{p_{1} \Rightarrow\right\}\right\rangle / \triangleright p_{1} \Rightarrow \text { and }\left\{\Rightarrow p_{1}\right\} / \Rightarrow \triangleright p_{1}
$$

Applications of these rules have the form:

$$
\frac{\Gamma, \varphi \Rightarrow E}{\Gamma, \triangleright \varphi \Rightarrow E} \quad \frac{\Gamma \Rightarrow \varphi}{\Gamma \Rightarrow \triangleright \varphi}
$$

Weak Affirmation: Let the connective $\rightarrow$ be defined using the following rules:

$$
\left\langle\left\{p_{1} \Rightarrow\right\}, \emptyset\right\rangle / p_{1} \Rightarrow \text { and }\left\{\Rightarrow p_{1}\right\} / \Rightarrow p_{1}
$$

Applications of these rules have the form:

$$
\frac{\Gamma, \varphi \Rightarrow}{\Gamma, \downarrow \Rightarrow E} \quad \frac{\Gamma \Rightarrow \varphi}{\Gamma \Rightarrow \emptyset}
$$

Note that the left-introduction rule for $\rightarrow$ includes one hard negative premise, to which no right context can be added. As a result, $\bullet \varphi \varphi$ is not provable.

Bowen's connectives: In [7], Bowen introduced an extension of the basic intuitionistic calculus with two new intuitionistic connectives? He defined these connectives by the following canonical rules:

$$
\begin{gathered}
\left\langle\left\{p_{2} \Rightarrow p_{1}\right\}, \emptyset\right\rangle / p_{1} \not \subset p_{2} \Rightarrow \text { and }\left\{\left(p_{1} \Rightarrow\right),\left(\Rightarrow p_{2}\right)\right\} / \Rightarrow p_{1} \not \subset p_{2} \\
\left\langle\left\{\left(\Rightarrow p_{1}\right),\left(\Rightarrow p_{2}\right)\right\}, \emptyset\right\rangle / p_{1} \mid p_{2} \Rightarrow \text { and }\left\{p_{1} \Rightarrow\right\} / \Rightarrow p_{1}\left|p_{2} \quad\left\{p_{2} \Rightarrow\right\} / \Rightarrow p_{1}\right| p_{2}
\end{gathered}
$$

Applications of these rules have the form:

$$
\begin{array}{cl}
\frac{\Gamma, \psi \Rightarrow \varphi}{\Gamma, \varphi \not \subset \psi \Rightarrow E} & \frac{\Gamma, \varphi \Rightarrow}{\Gamma \Rightarrow \varphi \not \subset \psi} \\
\frac{\Gamma \Rightarrow \varphi \quad \Gamma \Rightarrow \psi}{\Gamma, \varphi \mid \psi \Rightarrow E} & \frac{\Gamma, \varphi \Rightarrow}{\Gamma \Rightarrow \varphi \mid \psi} \frac{\Gamma, \psi \Rightarrow}{\Gamma \Rightarrow \varphi \mid \psi}
\end{array}
$$

Definition 3.5. A non-strict single-conclusion sequent system is called canonical if it satisfies the following conditions:

(1) Its axioms are the sequents of the form $\varphi \Rightarrow \varphi$.

(2) Weakening and cut are among its rules. Applications of these rules have the form:

$$
\frac{\Gamma \Rightarrow E}{\Gamma, \Delta \Rightarrow E} \frac{\Gamma \Rightarrow}{\Gamma \Rightarrow \psi} \quad \frac{\Gamma \Rightarrow \varphi \quad \Delta, \varphi \Rightarrow E}{\Gamma, \Delta \Rightarrow E}
$$

(3) Each of its other rules is either a canonical right-introduction rule or a canonical leftintroduction rule.

Convention. From now on, by canonical system we shall mean "non-strict single-conclusion canonical system".

\footnotetext{
${ }^{2}$ He also presented "neither-nor" connective, which we do not describe here, since this connective can be expressed by the four basic intuitionistic connectives.
} 
Definition 3.6. Let $\mathbf{G}$ be a canonical system, and let $\mathcal{S} \cup\{s\}$ be a set of sequents. $\mathcal{S} \vdash_{\mathbf{G}}^{\text {seq }} s$ iff there exists a derivation in $\mathbf{G}$ of $s$ from $\mathcal{S}$. The sequents of $\mathcal{S}$ are called the assumptions (or non-logical axioms) of such a derivation.

Definition 3.7. The etcr $\vdash_{\mathbf{G}}$ which is induced by a canonical system $\mathbf{G}$ is defined by: $\mathcal{T} \vdash_{\mathbf{G}} E$ iff there exists a finite $\Gamma \subseteq \mathcal{T}$ such that $\vdash_{\mathbf{G}}^{\text {seq }} \Gamma \Rightarrow E$.

Proposition 3.8. $\vdash_{\mathbf{G}}$ is a structural and finitary etcr for every canonical system $\mathbf{G}$.

Proposition 3.9. $\mathcal{T} \vdash_{\mathbf{G}} E$ iff $\{\Rightarrow \psi \mid \psi \in \mathcal{T}\} \vdash_{\mathbf{G}}^{\text {seq }} \Rightarrow E$.

We leave the easy proofs of the last two propositions to the reader.

\section{Consistency and Coherence}

Consistency (or non-triviality) is a crucial property of a deductive system. The goal of this section is to find a constructive criterion for it in the framework of canonical systems.

Definition 4.1. A canonical system $\mathbf{G}$ is called consistent iff $\nvdash_{\mathbf{G}}^{\text {seq }} p_{1} \Rightarrow p_{2}$.

Proposition 4.2. A canonical system $\mathbf{G}$ is consistent iff $\vdash_{\mathbf{G}}$ is consistent.

In multiple-conclusion canonical systems ([3]), as well as in strict single-conclusion canonical systems (4]), consistency is equivalent to coherence. Roughly speaking, a coherent system is a system in which the rules cannot lead to new conflicts: the conclusions of two rules can contradict each other only if their joint set of premises is already inconsistent. Next we adapt this criterion to the present case:

Definition 4.3. A set $\mathcal{R}$ of canonical rules for an $n$-ary connective $\diamond$ is called coherent iff $S_{1} \cup S_{2} \cup S_{3}$ is classically inconsistent whenever $\mathcal{R}$ contains both $\left\langle S_{1}, S_{2}\right\rangle / \diamond\left(p_{1}, \ldots, p_{n}\right) \Rightarrow$ and $S_{3} / \Rightarrow \diamond\left(p_{1}, \ldots, p_{n}\right)$.

Remark 4.4. It is known that a set of clauses is classically inconsistent iff the empty clause can be derived from it using only cuts.

Example 4.5. Every connective introduced in Example 3.4, has a coherent set of rules. For example, for the two rules for implication we have $S_{1}=\left\{\Rightarrow p_{1}\right\}, S_{2}=\left\{p_{2} \Rightarrow\right\}, S_{3}=$ $\left\{p_{1} \Rightarrow p_{2}\right\}$, and $S_{1} \cup S_{2} \cup S_{3}$ is the classically inconsistent set $\left\{\left(\Rightarrow p_{1}\right),\left(p_{2} \Rightarrow\right),\left(p_{1} \Rightarrow p_{2}\right)\right\}$ (from which the empty sequent can be derived using two cuts). For the two rules for semiimplication we have $S_{1}=\left\{\Rightarrow p_{1}\right\}, S_{2}=\left\{p_{2} \Rightarrow\right\}, S_{3}=\left\{\Rightarrow p_{2}\right\}$, and $S_{1} \cup S_{2} \cup S_{3}$ is the classically inconsistent set $\left\{\left(\Rightarrow p_{1}\right),\left(p_{2} \Rightarrow\right),\left(\Rightarrow p_{2}\right)\right\}$ (from which the empty sequent can be derived using one cut).

Definition 4.6. A canonical system $\mathbf{G}$ is called coherent iff for each connective $\diamond$, the set of rules in $\mathbf{G}$ for $\diamond$ is coherent.

Unfortunately, the next example shows that in the present case coherence is not necessary for consistency.

Example 4.7. Let $\mathbf{G}$ be a canonical system for a language which includes a single unary connective $\circ$, having the following rules:

$$
\left\langle\emptyset,\left\{p_{1} \Rightarrow\right\}\right\rangle / \circ p_{1} \Rightarrow \text { and }\left\{p_{1} \Rightarrow\right\} / \Rightarrow \circ p_{1}
$$


Applications of these rules have the form:

$$
\frac{\Gamma, \varphi \Rightarrow E}{\Gamma, \circ \varphi \Rightarrow E} \quad \frac{\Gamma, \varphi \Rightarrow}{\Gamma \Rightarrow \circ \varphi}
$$

Obviously, $\mathbf{G}$ is not coherent. However, it can easily be proved (using induction) that the only sequents provable in $\mathbf{G}$ from no assumptions are the sequents of the form $\Gamma \Rightarrow \psi$, where $\circ^{n} \psi \in \Gamma$ for some $n \geq 0$ (here $\circ^{0} \psi=\psi$ and $\circ^{n+1} \psi=\circ \circ^{n} \psi$ ). In particular, $p_{1} \Rightarrow p_{2}$ is not provable in $\mathbf{G}$ from no assumptions, and so $\mathbf{G}$ is consistent.

To overcome this difficulty, we define a stronger notion of consistency, and show that in the context of non-strict canonical systems, the coherence criterion is equivalent to this stronger notion.

Definition 4.8. A canonical system $\mathbf{G}$ is called strongly consistent iff $\left(\Rightarrow p_{1}\right),\left(p_{2} \Rightarrow\right) \nvdash_{\mathbf{G}}^{\text {seq }} \Rightarrow$.

Proposition 4.9. Every strongly consistent canonical system is also consistent.

Proof. Let $\mathbf{G}$ be an inconsistent canonical system. Then $\underset{\mathbf{G}}{\vdash_{\mathbf{G}}^{\text {seq }}} p_{1} \Rightarrow p_{2}$. Using the assumptions $\left(\Rightarrow p_{1}\right),\left(p_{2} \Rightarrow\right)$ and two cuts we get $\left(\Rightarrow p_{1}\right),\left(p_{2} \Rightarrow\right) \vdash_{\mathbf{G}}^{s e q} \Rightarrow$.

The following derivation shows that the system from Example 4.7 is not strongly consistent, and so strong consistency is indeed strictly stronger than consistency.

$$
\frac{p_{1} \Rightarrow \frac{\frac{p_{1} \Rightarrow p_{1}}{\circ p_{1} \Rightarrow p_{1}} \circ \Rightarrow \frac{p_{1} \Rightarrow}{\Rightarrow \circ p_{1}}}{\Rightarrow p_{1}} \text { cut } \text { cut }}{\Rightarrow}
$$

We note that strong consistency is a very natural demand from a system: in strongly inconsistent systems it suffices to have one provable sequent of the form $\psi \Rightarrow$, and one provable sequent of the form $\Rightarrow \varphi$, to make every sequent provable.

Theorem 4.10. Every strongly consistent canonical system is coherent.

Proof. Let $\mathbf{G}$ be an incoherent canonical system. This means that $\mathbf{G}$ includes two rules $\left\langle S_{1}, S_{2}\right\rangle / \diamond\left(p_{1}, \ldots, p_{n}\right) \Rightarrow$ and $S_{3} / \Rightarrow \diamond\left(p_{1}, \ldots, p_{n}\right)$, such that the set of clauses $S_{1} \cup S_{2} \cup S_{3}$ is classically satisfiable. Let $v$ be an assignment in $\{t, f\}$ that satisfies all the clauses in $S_{1} \cup S_{2} \cup S_{3}$. Define a substitution $\sigma$ by:

$$
\sigma(p)= \begin{cases}p_{1} & v(p)=t \\ p_{2} & v(p)=f\end{cases}
$$

Since $v$ satisfies all the clauses in $S_{1} \cup S_{2} \cup S_{3}$, for every $\Pi \Rightarrow E \in S_{1} \cup S_{2} \cup S_{3}$ we have $p_{2} \in \sigma(\Pi)$ or $p_{1} \in \sigma(E)$. Hence, every element of $\sigma\left(S_{1} \cup S_{2} \cup S_{3}\right)$ can be derived from $\left(\Rightarrow p_{1}\right),\left(p_{2} \Rightarrow\right)$ by weakening. Now by applying the rules $\left\langle S_{1}, S_{2}\right\rangle / \diamond\left(p_{1}, \ldots, p_{n}\right) \Rightarrow$ and $S_{3} / \Rightarrow \diamond\left(p_{1}, \ldots, p_{n}\right)$ to these sequents we get proofs from $\left(\Rightarrow p_{1}\right),\left(p_{2} \Rightarrow\right)$ of the sequents $\Rightarrow \sigma\left(\diamond\left(p_{1}, \ldots, p_{n}\right)\right)$ and $\sigma\left(\diamond\left(p_{1}, \ldots, p_{n}\right)\right) \Rightarrow$. That $\left(\Rightarrow p_{1}\right),\left(p_{2} \Rightarrow\right) \vdash_{\mathbf{G}}^{\text {seq }} \Rightarrow$ then follows using a cut. 
The last theorem implies that coherence is a necessary demand from any acceptable canonical system G. In the sequel (Corollary 6.10) we show that coherence is also sufficient to ensure strong consistency.

Remark 4.11. Our coherence criterion can be proved to be equivalent (for fully-structural sequent systems) to the reductivity criterion defined in [8]. However, in the framework of [8] a connective essentially has infinitely many introduction rules, while our framework makes it possible to convert these infinite sets of rules into finite ones.

\section{Semantics for Canonical Systems}

In this section we generalize Kripke semantics for intuitionistic logic to arbitrary coherent canonical systems. For this we use non-deterministic Kripke frames and semiframes.

Definition 5.1. Let $\langle W, \leq\rangle$ be a nonempty partially ordered set. Let $\mathcal{U}$ be a set of formulas. A function $v: W \times \mathcal{U} \rightarrow\{t, f\}$ is called persistent iff for every $a \in W$ and $\varphi \in \mathcal{U}, v(a, \varphi)=t$ implies that $v(b, \varphi)=t$ for every $b \in W$ such that $a \leq b$.

Definition 5.2. Let $\mathcal{U}$ be a set of formulas closed under subformulas. A $\mathcal{U}$-semiframe is a triple $\mathcal{W}=\langle W, \leq, v\rangle$ such that:

(1) $\langle W, \leq\rangle$ is a nonempty partially ordered set.

(2) $v$ is a persistent function from $W \times \mathcal{U}$ to $\{t, f\}$.

When $\mathcal{U}=\mathcal{F}$ a $\mathcal{U}$-semiframe is also called an $\mathcal{L}$-frame.

Remark 5.3. To understand the need to consider semiframes, we note that to be useful and effective, a denotational semantics of a propositional logic should be analytic. This means that in order to determine whether a sequent $s$ follows from a set $\mathcal{S}$ of sequents, it should be sufficient to consider partial valuations, defined only on the set of subformulas of the formulas in $\mathcal{S} \cup\{s\}$. In the present case, such partial valuations are provided by semiframes.

Definition 5.4. Let $\mathcal{W}=\langle W, \leq, v\rangle$ be a $\mathcal{U}$-semiframe.

(1) A sequent $\Gamma \Rightarrow E$ is locally true in $a \in W$ iff $\Gamma \cup E \subseteq \mathcal{U}$, and either $v(a, \psi)=f$ for some $\psi \in \Gamma$, or $E=\{\varphi\}$ and $v(a, \varphi)=t$.

(2) A sequent is true (or absolutely true) in $a \in W$ iff it is locally true in every $b \geq a$.

(3) $\mathcal{W}$ is a model of a sequent $s$ iff $s$ is true in every $a \in W$ (equivalently, if $s$ is locally true in every $a \in W)$. It is a model of a set $\mathcal{S}$ of sequents if it is a model of every $s \in \mathcal{S}$.

(4) $\mathcal{W}$ is a model of a formula $\varphi$ iff $v(a, \varphi)=t$ for every $a \in W$. It is a model of a theory $\mathcal{T}$ if it is a model of every $\varphi \in \mathcal{T}$.

Remark 5.5. From the point of view of local truth, a sequent is understood according to its classical interpretation as a disjunction (either one of the formulas in its left side is "false" or its right side is "true"). On the other hand, the notion of absolute truth is based on viewing a sequent as expressing a real (constructive) entailment between its two sides. Note that because of the persistence condition, for sequents of the form $\Rightarrow \varphi$ there is no difference between local truth in $a$ or absolute truth in $a$. Obviously, $\mathcal{W}$ is a model of such a sequent iff it is a model of $\varphi$. 
Persistence is the only general condition which is satisfied by the semantics of every coherent canonical system. In addition, to every specific canonical system corresponds a set of constraints which are directly related to its set of canonical rules. The idea is that a canonical rule for a connective $\diamond$ imposes restrictions on the truth-values that can be assigned to $\diamond$-formulas. Next we describe these restrictions.

Definition 5.6. Let $\mathcal{W}=\langle W, \leq, v\rangle$ be a $\mathcal{U}$-semiframe.

(1) An $\mathcal{L}$-substitution $\sigma$ (locally) satisfies a sequent $\Gamma \Rightarrow E$ in $a \in W$ iff $\sigma(\Gamma) \Rightarrow \sigma(E)$ is (locally) true in ${ }^{3}$.

(2) An $\mathcal{L}$-substitution fulfils a canonical right-introduction rule in $a \in W$ (with respect to $\mathcal{W})$ iff it satisfies in a every premise of the rule.

(3) An $\mathcal{L}$-substitution fulfils a canonical left-introduction rule in $a \in W$ (with respect to $\mathcal{W}$ ) iff it satisfies in $a$ every hard premise of the rule, and locally satisfies in $a$ every soft premise of the rule.

(4) Let $r$ be a canonical rule for an $n$-ary connective $\diamond$. $\mathcal{W}$ respects $r$ iff for every $a \in W$ and every substitution $\sigma$ : if $\sigma$ fulfils $r$ in $a$ and $\sigma\left(\diamond\left(p_{1}, \ldots, p_{n}\right)\right) \in \mathcal{U}$ then $\sigma$ locally satisfies conclusion of $r$ in $a$.

Note that absolute truth is used for premises of right introduction rules, as well as for hard premises of left introduction rules. Local truth is used only for soft premises of left introduction rule. This is the main difference between this semantics and the one described in [4] for the strict framework. In [4, the difference between absolute and local truth corresponds to the syntactic distinction between definite and negative sequents (absolute truth is used for definite premises, and local truth is used for negative premises). In the present case, since negative sequents may also serve as premises of right introduction rules and as hard premises of left introduction rules, this syntactic distinction is irrelevant for the semantics definition.

Remark 5.7. Because of the persistence condition, a definite sequent of the form $\Rightarrow \psi$ is satisfied in $a$ by $\sigma$ iff $v(a, \sigma(\psi))=t$.

Examples 5.8. We describe the semantic effects of some rules from Example 3.4 ,

Negation: An $\mathcal{L}$-frame $\mathcal{W}=\langle W, \leq, v\rangle$ respects the rule $(\neg \Rightarrow)$ if $v(a, \neg \psi)=f$ whenever $v(a, \psi)=t$. Because of the persistence condition, if $v(b, \neg \psi)=f$ for some $b \geq a$ then $v(a, \neg \psi)=f$. And so, $\mathcal{W}$ respects $(\neg \Rightarrow)$ if $v(a, \neg \psi)=f$ whenever $v(b, \psi)=t$ for some $b \geq a$. It respects $(\Rightarrow \neg)$ if $v(a, \neg \psi)=t$ whenever $v(b, \psi)=f$ for every $b \geq a$. Hence the two rules together impose exactly the well-known Kripke semantics for intuitionistic negation.

Implication: An $\mathcal{L}$-frame $\mathcal{W}=\langle W, \leq, v\rangle$ respects the rule $(\supset \Rightarrow)$ iff for every $a \in W$, $v(a, \varphi \supset \psi)=f$ whenever $v(b, \varphi)=t$ for every $b \geq a$ and $v(a, \psi)=f$ (the latter because $\psi \Rightarrow$ is an instance of a soft premise). Because of the persistence condition, this is equivalent to $v(a, \varphi \supset \psi)=f$ whenever $v(a, \varphi)=t$ and $v(a, \psi)=f$. Again by the persistence condition, $v(a, \varphi \supset \psi)=f$ iff $v(b, \varphi \supset \psi)=f$ for some $b \geq a$. Hence, we get: $v(a, \varphi \supset \psi)=f$ whenever there exists $b \geq a$ such that $v(b, \varphi)=t$ and $v(b, \psi)=f$. $\mathcal{W}$ respects $(\Rightarrow \supset)$ iff for every $a \in W, v(a, \varphi \supset \psi)=t$ whenever for every $b \geq a$, either $v(b, \varphi)=f$ or $v(b, \psi)=t$. Hence the two rules together impose exactly the well-known Kripke semantics for intuitionistic implication ([1] $)$. It is easy to verify that the same applies to conjunction and disjunction, using the usual rules for these connectives.

\footnotetext{
${ }^{3}$ When $E=\emptyset$, recall that $\sigma(\emptyset)=\emptyset$.
} 
Semi-Implication: An $\mathcal{L}$-frame $\mathcal{W}=\langle W, \leq, v\rangle$ respects the rule $(\leadsto \Rightarrow)$ under the same conditions it respects $(\supset \Rightarrow)$. $\mathcal{W}$ respects $(\Rightarrow \leadsto)$ iff for every $a \in W, v(a, \varphi \leadsto \psi)=t$ whenever $v(a, \psi)=t$ (recall that this is equivalent to $v(b, \psi)=t$ for every $b \geq a$ ). Note that in this case the two rules for $\sim$ do not always determine the value assigned to $\varphi \leadsto \psi$ : if $v(a, \psi)=f$, and there is no $b \geq a$ such that $v(b, \varphi)=t$ and $v(b, \psi)=f$, then $v(a, \varphi \leadsto \psi)$ is free to be either $t$ or $f$. So the semantics of this connective is non-deterministic.

Converse Non-Implication: An $\mathcal{L}$-frame $\mathcal{W}=\langle W, \leq, v\rangle$ respects the rule $(\not \subset \Rightarrow)$ provided that $v(a, \varphi \not \subset \psi)=f$ whenever for every $b \geq a$ either $v(b, \varphi)=t$ or $v(b, \psi)=f$. Because of the persistence condition, this is equivalent to $v(a, \varphi \not \subset \psi)=f$ if either there exists some $b \geq a$ such that $v(b, \varphi)=t$, or if $v(b, \psi)=f$ for every $b \geq a$. It respects $(\Rightarrow \not \subset)$ if $v(a, \varphi \not \subset \psi)=t$ whenever $v(b, \varphi)=f$ and $v(b, \psi)=t$ for every $b \geq a$. Because of the persistence condition, this is equivalent to $v(a, \varphi \not \subset \psi)=t$ whenever $v(a, \psi)=t$ and $v(b, \varphi)=f$ for every $b \geq a$. This implies that $v(a, \varphi \not \subset \psi)$ is free when $v(b, \varphi)=f$ for every $b \geq a, v(a, \psi)=f$, and there exists $b \geq a$ such that $v(b, \psi)=t$. For example, consider the following two $\left\{p_{1}, p_{2}, p_{1} \not \subset p_{2}\right\}$-semiframes:
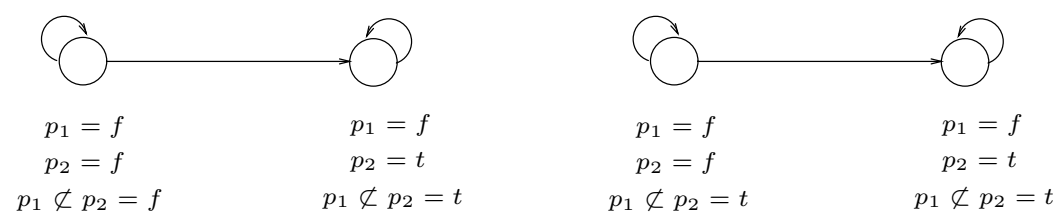

While there is no difference between these two semi-frames with respect to atomic formulas, the truth-values assigned to $p_{1} \not \subset p_{2}$ in one of their two worlds are different. Now both semiframes respect the two rules of $\not \subset$. Hence the semantics of this connective is non-deterministic 4

Not Both: An $\mathcal{L}$-frame $\mathcal{W}=\langle W, \leq, v\rangle$ respects the rule $(\mid \Rightarrow)$ if $v(a, \varphi \mid \psi)=f$ whenever $v(b, \varphi)=t$ and $v(b, \psi)=t$ for every $b \geq a$. Because of the persistence condition, this is equivalent to $v(a, \varphi \mid \psi)=f$ whenever $v(b, \psi)=v(b, \varphi)=t$ for some $b \geq a$. It respects $(\Rightarrow \mid)_{1}$ if $v(a, \varphi \mid \psi)=t$ whenever $v(b, \varphi)=f$ for every $b \geq a$. It respects $(\Rightarrow \mid)_{2}$ if $v(a, \varphi \mid \psi)=t$ whenever $v(b, \psi)=f$ for every $b \geq a$. This implies that $v(a, \varphi \mid \psi)$ is free when there exist $b_{1}, b_{2} \geq a$ such that $v\left(b_{1}, \varphi\right)=v\left(b_{2}, \psi\right)=t$, but there does not exist $b \geq a$ such that $v(b, \varphi)=v(b, \psi)=t$ (this is possible because the order relation does not have to be linear). Again, the induced semantics is non-deterministic.

Affirmation: An $\mathcal{L}$-frame $\mathcal{W}=\langle W, \leq, v\rangle$ respects the rule $(\triangleright \Rightarrow)$ if $v(a, \triangleright \psi)=f$ whenever $v(a, \psi)=f$. It respects $(\Rightarrow \triangleright)$ if $v(a, \triangleright \psi)=t$ whenever $v(a, \psi)=t$. This means that for every $a \in W, v(a, \triangleright \psi)$ simply equals $v(a, \psi)$.

Weak Affirmation: An $\mathcal{L}$-frame $\mathcal{W}=\langle W, \leq, v\rangle$ respects the rule $(\gg)$ if $v(a, \downarrow \psi)=f$ whenever $v(b, \psi)=f$ for every $b \geq a$. It respects $(\Rightarrow)$ if $v(a, \psi)=t$ whenever $v(b, \psi)=t$ for every $b \geq a$. Because of the persistence condition, this is equivalent to $v(a, \psi)=t$ whenever $v(a, \psi)=t$. This implies that $v(a, \psi)$ is free when $v(a, \psi)=f$ and $v(b, \psi)=t$ for some $b \geq a$. Again, we obtain non-deterministic semantics.

Definition 5.9. Let $\mathbf{G}$ be a canonical system. A $\mathcal{U}$-semiframe is $\mathbf{G}$-legal iff it respects all the canonical rules of $\mathbf{G}$.

\footnotetext{
${ }^{4}$ Note that no semantic characterizations for "converse non-implication" and "not both" were presented in [7, where these connectives were first introduced.
} 
We can now give the definition of the semantic relations induced by a canonical system:

Definition 5.10. Let $\mathbf{G}$ be a coherent canonical system, and let $\mathcal{S} \cup\{s\}$ be a set of sequents. $\mathcal{S} \vDash_{\mathrm{G}}^{\text {seq }} s$ iff every $\mathbf{G}$-legal $\mathcal{L}$-frame which is a model of $\mathcal{S}$ is also a model of $s$.

Definition 5.11. Let $\mathbf{G}$ be a coherent canonical system. The semantic etcr $\vDash_{\mathbf{G}}$ between formulas which is induced by $\mathbf{G}$ is defined by: $\mathcal{T} \vDash_{\mathbf{G}} E$ iff every $\mathbf{G}$-legal $\mathcal{L}$-frame which is a model of $\mathcal{T}$ is also a model of $E$.

Again we have:

Proposition 5.12. $\mathcal{T} \vDash_{\mathbf{G}} E$ iff $\{\Rightarrow \psi \mid \psi \in \mathcal{T}\} \vDash_{\mathbf{G}}^{s e q} \Rightarrow E$.

\section{Soundness, Completeness, Cut-elimination}

In this section we show that the syntactic and semantic consequence relations between sequents which are induced by a given coherent canonical system are identical. In addition, we present a semantic proof of cut-elimination for arbitrary coherent canonical systems. There are a lot of similarities between the proofs of this section and the corresponding proofs in [4]. However, the proofs in [4] correspond to different definitions, and so, for the sake of completeness, we include here the full proofs.

Theorem 6.1. Every coherent canonical system $\mathbf{G}$ is strongly sound with respect to the semantics of $\mathbf{G}$-legal frames. In other words: If $\mathcal{S} \vdash_{\mathbf{G}}^{\text {seq }}$ s then $\mathcal{S} \models_{\mathbf{G}}^{\text {seq }}$ s.

Proof. Assume that $\mathcal{S} \vdash_{\mathbf{G}}^{\text {seq }} s$, and $\mathcal{W}=\langle W, \leq, v\rangle$ is a $\mathbf{G}$-legal model of $\mathcal{S}$. We show that $s$ is locally true in every $a \in W$. Since the axioms of $\mathbf{G}$ and the assumptions of $\mathcal{S}$ trivially have this property, and the cut and weakening rules obviously preserve it, it suffices to show that the property of being locally true in every $a \in W$ is also preserved by applications of the logical rules of $\mathbf{G}$.

- Suppose $\Gamma \Rightarrow \sigma\left(\diamond\left(p_{1}, \ldots, p_{n}\right)\right)$ is derived from $\left\{\Gamma, \sigma\left(\Pi_{i}\right) \Rightarrow \sigma\left(E_{i}\right)\right\}_{1 \leq i \leq m}$ using the rule $r=\left\{\Pi_{i} \Rightarrow E_{i}\right\}_{1 \leq i \leq m} / \Rightarrow \diamond\left(p_{1}, \ldots, p_{n}\right)$. Assume that all the premises of this application have the required property. We show that so does its conclusion. Let $a \in W$. If $v(a, \psi)=f$ for some $\psi \in \Gamma$, then obviously $\Gamma \Rightarrow \sigma\left(\diamond\left(p_{1}, \ldots, p_{n}\right)\right)$ is locally true in $a$. Assume otherwise. Then the persistence condition implies that $v(b, \psi)=t$ for every $\psi \in \Gamma$ and $b \geq a$. Thus our assumption concerning the sequents $\left\{\Gamma, \sigma\left(\Pi_{i}\right) \Rightarrow \sigma\left(E_{i}\right)\right\}_{1 \leq i \leq m}$ entails that for every $b \geq a$ and $1 \leq i \leq m$, either $v(b, \psi)=f$ for some $\psi \in \sigma\left(\Pi_{i}\right)$, or $E_{i}=\left\{q_{i}\right\}$ (i.e. $E_{i}$ is not empty) and $v\left(b, \sigma\left(q_{i}\right)\right)=t$. It follows that for $1 \leq i \leq m$, $\Pi_{i} \Rightarrow E_{i}$ is satisfied in $a$ by $\sigma$. Thus, $\sigma$ fulfils $r$ in $a$. Since $\mathcal{W}$ respects $r$, it follows that $v\left(a, \sigma\left(\diamond\left(p_{1}, \ldots, p_{n}\right)\right)\right)=t$.

- Now we deal with left-introduction rules. Suppose $\Gamma, \sigma\left(\diamond\left(p_{1}, \ldots, p_{n}\right)\right) \Rightarrow E$ is derived from $\left\{\Gamma, \sigma\left(\Pi_{i}\right) \Rightarrow \sigma\left(E_{i}\right)\right\}_{1 \leq i \leq m}$ and $\left\{\Gamma, \sigma\left(\Sigma_{i}\right) \Rightarrow E\right\}_{1 \leq i \leq k}$, using the left-introduction rule $r=\left\langle\left\{\Pi_{i} \Rightarrow E_{i}\right\}_{1 \leq i \leq m},\left\{\Sigma_{i} \Rightarrow\right\}_{1 \leq i \leq k}\right\rangle / \diamond\left(p_{1}, \ldots, p_{n}\right) \Rightarrow$. Assume that all the premises of this application have the required property. We show that so does its conclusion. Let $a \in W$. If $v(a, \psi)=f$ for some $\psi \in \Gamma$ or $E=\{\theta\}$ and $v(a, \theta)=t$, then we are done. Assume otherwise. Then $E$ is either empty or $E=\{\theta\}$ and $v(a, \theta)=f$, and (by the persistence condition) $v(b, \psi)=t$ for every $\psi \in \Gamma$ and $b \geq a$. Thus our assumption concerning the sequents $\left\{\Gamma, \sigma\left(\Pi_{i}\right) \Rightarrow \sigma\left(E_{i}\right)\right\}_{1 \leq i \leq m}$ entails that for every $b \geq a$ and $1 \leq i \leq m$, either $v(b, \psi)=f$ for some $\psi \in \sigma\left(\Pi_{i}\right)$, or $E_{i}=\left\{q_{i}\right\}$ and $v\left(b, \sigma\left(q_{i}\right)\right)=t$. This immediately implies that the hard premises of $r$ are satisfied in $a$ by $\sigma$. Since $E$ is either empty or 
$E=\{\theta\}$ and $v(a, \theta)=f$, our assumption concerning $\left\{\Gamma, \sigma\left(\Sigma_{i}\right) \Rightarrow E\right\}_{1 \leq i \leq k}$ entails that for every $1 \leq i \leq k, v(a, \psi)=f$ for some $\psi \in \sigma\left(\Sigma_{i}\right)$. Hence the soft premises of $r$ are locally satisfied in $a$ by $\sigma$. Thus, $\sigma$ fulfils $r$ in $a$. Since $\mathcal{W}$ respects $r$, it follows that $v\left(a, \sigma\left(\diamond\left(p_{1}, \ldots, p_{n}\right)\right)\right)=f$.

For the converse, we define $\mathcal{S}$-proofs and prove the following key result.

Definition 6.2. Let $\mathcal{S}$ be sets of sequents. A proof $P$ in a canonical system is called an $\mathcal{S}$-proof iff the cut formula of every cut in $P$ occurs in $\mathcal{S}$.

Theorem 6.3. Let $\mathbf{G}$ be a coherent canonical system in $\mathcal{L}$, and let $\mathcal{S} \cup\{s\}$ be a set of sequents in $\mathcal{L}$. Then either there is an $\mathcal{S}$-proof of $s$ from $\mathcal{S}$ in $\mathbf{G}$, or there is a $\mathbf{G}$-legal $\mathcal{L}$-frame which is model of $\mathcal{S}$, but not a model of $s$.

Proof. Assume that $s=\Gamma_{0} \Rightarrow E_{0}$ does not have an $\mathcal{S}$-proof from $\mathcal{S}$ in $\mathbf{G}$. We construct a G-legal $\mathcal{L}$-frame $\mathcal{W}$ which is a model of $\mathcal{S}$ but not of $s$. Let $\mathcal{U}$ be the set of subformulas of $\mathcal{S} \cup\{s\}$. Given a subset $E$ of $\mathcal{U}$ which is either a singleton or empty, call a theory $\mathcal{T} \subseteq \mathcal{U}$ $E$-maximal if there is no finite $\Gamma \subseteq \mathcal{T}$ such that $\Gamma \Rightarrow E$ has an $\mathcal{S}$-proof from $\mathcal{S}$, but every proper extension $\mathcal{T}^{\prime} \subseteq \mathcal{U}$ of $\mathcal{T}$ contains such a finite subset $\Gamma$. Obviously, if $\Gamma \cup E \subseteq \mathcal{U}$ and $\Gamma \Rightarrow E$ has no $\mathcal{S}$-proof from $\mathcal{S}$, then $\Gamma$ can be extended to a theory $\mathcal{T} \subseteq \mathcal{U}$ which is $E$-maximal. In particular: $\Gamma_{0}$ can be extended to a $E_{0}$-maximal theory $\mathcal{T}_{0}$.

Now let $\mathcal{W}=\langle W, \subseteq, v\rangle$, where:

- $W$ is the set of all extensions of $\mathcal{T}_{0}$ in $\mathcal{U}$ which are $E$-maximal for some $E \subseteq \mathcal{U}$ (recall that $E$ is either singleton or empty).

- $v$ is defined inductively as follows. For atomic formulas:

$$
v(\mathcal{T}, p)= \begin{cases}t & p \in \mathcal{T} \\ f & p \notin \mathcal{T}\end{cases}
$$

Suppose $v\left(\mathcal{T}, \psi_{i}\right)$ has been defined for every $\mathcal{T} \in W$ and $1 \leq i \leq n$.

We let $v\left(\mathcal{T}, \diamond\left(\psi_{1}, \ldots, \psi_{n}\right)\right)=t$ iff at least one of the following holds with respect to the semiframe constructed so far:

(1) There exists a right-introduction rule for $\diamond$ which is fulfilled in $\mathcal{T}$ by a substitution $\sigma$ such that $\sigma\left(p_{i}\right)=\psi_{i}(1 \leq i \leq n)$.

$(2) \diamond\left(\psi_{1}, \ldots, \psi_{n}\right) \in \mathcal{T}$, and there do not exist $\mathcal{T}^{\prime} \in W$ and a left-introduction rule $r$ for $\diamond$, such that $\mathcal{T} \subseteq \mathcal{T}^{\prime}$, and $r$ is fulfilled in $\mathcal{T}^{\prime}$ by a substitution $\sigma$ such that $\sigma\left(p_{i}\right)=\psi_{i}$ $(1 \leq i \leq n)$.

First we prove that $\mathcal{W}$ is an $\mathcal{L}$-frame:

- $W$ is not empty because $\mathcal{T}_{0} \in W$.

- We prove by structural induction that $v$ is persistent:

For atomic formulas $v$ is trivially persistent since the order is $\subseteq$.

Assume that $v$ is persistent for $\psi_{1}, \ldots, \psi_{n}$. We prove its persistence for $\diamond\left(\psi_{1}, \ldots, \psi_{n}\right)$. So assume that $v\left(\mathcal{T}, \diamond\left(\psi_{1}, \ldots, \psi_{n}\right)\right)=t$ and $\mathcal{T} \subseteq \mathcal{T}^{*}$. By the definition of $v$ there are two possibilities:

(1) There exists a right-introduction rule for $\diamond$ which is fulfilled in $\mathcal{T}$ by a substitution $\sigma$ such that $\sigma\left(p_{i}\right)=\psi_{i}(1 \leq i \leq n)$. This is also trivially true in $\mathcal{T}^{*}$, and so $v\left(\mathcal{T}^{*}, \diamond\left(\psi_{1}, \ldots, \psi_{n}\right)\right)=t$. 
$(2) \diamond\left(\psi_{1}, \ldots, \psi_{n}\right) \in \mathcal{T}$, and there do not exist $\mathcal{T}^{\prime} \in W$ and a left-introduction rule $r$ for $\diamond$, such that $\mathcal{T} \subseteq \mathcal{T}^{\prime}$, and $r$ is fulfilled in $\mathcal{T}^{\prime}$ by a substitution $\sigma$ such that $\sigma\left(p_{i}\right)=\psi_{i}$ $(1 \leq i \leq n)$. Then $\diamond\left(\psi_{1}, \ldots, \psi_{n}\right) \in \mathcal{T}^{*}\left(\right.$ since $\left.\mathcal{T} \subseteq \mathcal{T}^{*}\right)$, and there cannot exist $\mathcal{T}^{\prime} \in W$ and a left-introduction rule $r$ for $\diamond$, such that $\mathcal{T}^{*} \subseteq \mathcal{T}^{\prime}$, and $r$ is fulfilled in $\mathcal{T}^{\prime}$ by such a substitution $\sigma$ (otherwise the same would hold for $\mathcal{T}$ ). Hence $v\left(\mathcal{T}^{*}, \diamond\left(\psi_{1}, \ldots, \psi_{n}\right)\right)=t$ in this case too.

Next we prove that $\mathcal{W}$ is $\mathbf{G}$-legal:

(1) The right-introduction rules are directly respected by the first condition in the definition of $v$.

(2) Let $r$ be a left-introduction rule for $\diamond$, and let $\mathcal{T} \in W$. Suppose that $r$ is fulfilled in $\mathcal{T}$ by a substitution $\sigma$, such that $\sigma\left(p_{i}\right)=\psi_{i}(1 \leq i \leq n)$. Then neither of the conditions under which $v\left(\mathcal{T}, \diamond\left(\psi_{1}, \ldots, \psi_{n}\right)\right)=t$ can hold:

(a) The second condition explicitly excludes the option that $r$ is fulfilled by $\sigma$ (in any $\mathcal{T}^{\prime} \in W$ such that $\mathcal{T} \subseteq \mathcal{T}^{\prime}$, including $\mathcal{T}$ itself).

(b) The first condition cannot be met because the coherence of $\mathbf{G}$ does not allow the sets of premises (of a right-introduction rule and a left-introduction rule for the same connective) to be locally satisfied together. Hence the two rules cannot be both fulfilled by the same substitution in the same element of $W$. To see this, assume by way of contradiction that $S_{1}$ and $S_{2}$ are the sets of premises of a leftintroduction rule for $\diamond, S_{3}$ is the set of premises of a right-introduction rule for $\diamond$, and there exists $\mathcal{T} \in W$ in which the three sets of premises are locally satisfied by a substitution $\sigma$ such that $\sigma\left(p_{i}\right)=\psi_{i}(1 \leq i \leq n)$. Let $u$ be an assignment in $\{t, f\}$ in which $u\left(p_{i}\right)=v\left(\mathcal{T}, \psi_{i}\right)$. Since $\sigma$ locally satisfies in $\mathcal{T}$ the three sets of premises, $u$ classically satisfies $S_{1}, S_{2}$ and $S_{3}$. This contradicts the coherence of $\mathbf{G}$.

It follows that $v\left(\mathcal{T}, \diamond\left(\psi_{1}, \ldots, \psi_{n}\right)\right)=f$, as required.

It remains to prove that $\mathcal{W}$ is a model of $\mathcal{S}$ but not of $s$. For this we first prove that the following hold for every $\mathcal{T} \in W$ and every formula $\psi \in \mathcal{U}$ :

(a): If $\psi \in \mathcal{T}$ then $v(\mathcal{T}, \psi)=t$.

(b): If $\mathcal{T}$ is $\{\psi\}$-maximal then $v(\mathcal{T}, \psi)=f$.

We prove (a) and (b) together by a simultaneous induction on the complexity of $\psi$. For atomic formulas they easily follow from the definition of $v$, and the fact that $p \Rightarrow p$ is an axiom. For the induction step, assume that (a) and (b) hold for $\psi_{1}, \ldots, \psi_{n} \in \mathcal{U}$. We prove them for $\diamond\left(\psi_{1}, \ldots, \psi_{n}\right) \in \mathcal{U}$.

- Assume that $\diamond\left(\psi_{1}, \ldots, \psi_{n}\right) \in \mathcal{T}$, but $v\left(\mathcal{T}, \diamond\left(\psi_{1}, \ldots, \psi_{n}\right)\right)=f$. By the definition of $v$, since $\diamond\left(\psi_{1}, \ldots, \psi_{n}\right) \in \mathcal{T}$ there should exist $\mathcal{T}^{\prime} \in W, \mathcal{T} \subseteq \mathcal{T}^{\prime}$, and a left-introduction rule, $r=\left\langle\left\{\Pi_{i} \Rightarrow E_{i}\right\}_{1 \leq i \leq m},\left\{\Sigma_{i} \Rightarrow\right\}_{1 \leq i \leq k}\right\rangle / \diamond\left(p_{1}, \ldots, p_{n}\right) \Rightarrow$, fulfilled in $\mathcal{T}^{\prime}$ by a substitution $\sigma$ such that $\sigma\left(p_{i}\right)=\psi_{i}(1 \leq i \leq n)$. As $\sigma$ locally satisfies in $\mathcal{T}^{\prime}$ every sequent in $\left\{\Sigma_{i} \Rightarrow\right\}_{1 \leq i \leq k}$, then for every $1 \leq i \leq k$ there exists $\psi_{j_{i}} \in \sigma\left(\Sigma_{i}\right)$ with $v\left(\mathcal{T}^{\prime}, \psi_{j_{i}}\right)=f$. By the induction hypothesis this implies that for every $1 \leq i \leq k$, there exists $\psi_{j_{i}} \in \sigma\left(\Sigma_{i}\right)$ such that $\psi_{j_{i}} \notin \mathcal{T}^{\prime}$. Let $E$ be the set for which $\mathcal{T}^{\prime}$ is maximal. Then for every $1 \leq i \leq k$ there is a finite $\Delta_{i} \subseteq \mathcal{T}^{\prime}$ such that $\Delta_{i}, \psi_{j_{i}} \Rightarrow E$ has an $\mathcal{S}$-proof from $\mathcal{S}$, and therefore $\Delta_{i}, \sigma\left(\Sigma_{i}\right) \Rightarrow E$ has such a proof. This in turn implies that there must exist $1 \leq i_{0} \leq m$ such that $\Gamma, \sigma\left(\Pi_{i_{0}}\right) \Rightarrow \sigma\left(E_{i_{0}}\right)$ has no $\mathcal{S}$-proof from $\mathcal{S}$ for any finite $\Gamma \subseteq \mathcal{T}^{\prime}$. Indeed, if such a proof exists for every $1 \leq i \leq m$, we would use the $k$ proofs of $\Delta_{i}, \sigma\left(\Sigma_{i}\right) \Rightarrow E$ for $1 \leq i \leq k$, the $m$ proofs for $\Gamma_{i}, \sigma\left(\Pi_{i}\right) \Rightarrow \sigma\left(E_{i}\right)$ for $1 \leq i \leq m$, some trivial weakenings, and the left-introduction rule 
$r$ to get an $\mathcal{S}$-proof from $\mathcal{S}$ of the sequent $\cup_{i=1}^{i=k} \Delta_{i}, \cup_{i=1}^{i=m} \Gamma_{i}, \diamond\left(\psi_{1}, \ldots, \psi_{n}\right) \Rightarrow E$. Since $\diamond\left(\psi_{1}, \ldots, \psi_{n}\right) \in \mathcal{T}$, this would contradict the $E$-maximality of $\mathcal{T}^{\prime}$. Using this $i_{0}$, extend $\mathcal{T}^{\prime} \cup \sigma\left(\Pi_{i_{0}}\right)$ to a $\sigma\left(E_{i_{0}}\right)$-maximal theory $\mathcal{T}^{\prime \prime}$. By the induction hypothesis, $v\left(\mathcal{T}^{\prime \prime}, \psi\right)=t$ for every $\psi \in \sigma\left(\Pi_{i_{0}}\right)$, and if $E_{i_{0}}=\{q\}$ (i.e. $E_{i_{0}}$ is not empty) then $v\left(\mathcal{T}^{\prime \prime}, \sigma(q)\right)=f$. Since $T^{\prime} \subseteq \mathcal{T}^{\prime \prime}$, this contradicts the fact that $\sigma$ satisfies $\Pi_{i_{0}} \Rightarrow E_{i_{0}}$ in $\mathcal{T}^{\prime}$.

- Assume that $\mathcal{T}$ is $\left\{\diamond\left(\psi_{1}, \ldots, \psi_{n}\right)\right\}$-maximal, but that $v\left(\mathcal{T}, \diamond\left(\psi_{1}, \ldots, \psi_{n}\right)\right)=t$. Obviously, $\diamond\left(\psi_{1}, \ldots, \psi_{n}\right) \notin \mathcal{T}$ (because $\diamond\left(\psi_{1}, \ldots, \psi_{n}\right) \Rightarrow \diamond\left(\psi_{1}, \ldots, \psi_{n}\right)$ is an axiom). Hence there exists a right-introduction rule, $r=\left\{\Pi_{i} \Rightarrow E_{i}\right\}_{1 \leq i \leq m} / \Rightarrow \diamond\left(p_{1}, \ldots, p_{n}\right)$, which is fulfilled in $\mathcal{T}$ by a substitution $\sigma$ such that $\sigma\left(p_{i}\right)=\psi_{i}(1 \leq i \leq n)$. As in the previous case, there must exist $1 \leq i_{0} \leq m$ such that $\Gamma, \sigma\left(\Pi_{i_{0}}\right) \Rightarrow \sigma\left(E_{i_{0}}\right)$ has no $\mathcal{S}$-proof from $\mathcal{S}$ for any finite $\Gamma \subseteq \mathcal{T}$ (if such a proof exists for every $1 \leq i \leq m$ with finite $\Gamma_{i} \subseteq \mathcal{T}$ than we could have an $\mathcal{S}$-proof from $\mathcal{S}$ of $\cup_{i=1}^{i=m} \Gamma_{i} \Rightarrow \diamond\left(\psi_{1}, \ldots, \psi_{n}\right)$ using the $m$ proofs of $\Gamma_{i}, \sigma\left(\Pi_{i}\right) \Rightarrow \sigma\left(E_{i}\right)$, some weakenings and $r$ ). Using this $i_{0}$, extend $\mathcal{T} \cup \sigma\left(\Pi_{i_{0}}\right)$ to a $\sigma\left(E_{i_{0}}\right)$-maximal theory $\mathcal{T}^{\prime}$. By the induction hypothesis $v\left(\mathcal{T}^{\prime}, \psi\right)=t$ for every $\psi \in \sigma\left(\Pi_{i_{0}}\right)$, and if $E_{i_{0}}=\{q\}$ (i.e. $E_{i_{0}}$ is not empty) then $v\left(\mathcal{T}^{\prime}, \sigma(q)\right)=f$. Since $\mathcal{T} \subseteq \mathcal{T}^{\prime}$, this contradicts the fact that $\sigma$ satisfies $\Pi_{i_{0}} \Rightarrow E_{i_{0}}$ in $\mathcal{T}$.

Next we note that (b) can be strengthened as follows:

(c) If $\psi \in \mathcal{U}, \mathcal{T} \in W$ and there is no finite $\Gamma \subseteq \mathcal{T}$ such that $\Gamma \Rightarrow \psi$ has an $\mathcal{S}$-proof from $\mathcal{S}$, then $v(\mathcal{T}, \psi)=f$.

Indeed, under these conditions $\mathcal{T}$ can be extended to a $\{\psi\}$-maximal theory $\mathcal{T}^{\prime}$. Now $\mathcal{T}^{\prime} \in W, \mathcal{T} \subseteq \mathcal{T}^{\prime}$, and by (b), $v\left(\mathcal{T}^{\prime}, \psi\right)=f$. Hence also $v(\mathcal{T}, \psi)=f$.

Now (a) and (b) together imply that $v\left(\mathcal{T}_{0}, \psi\right)=t$ for every $\psi \in \Gamma_{0} \subseteq \mathcal{T}_{0}$, and if $E_{0}=\{\theta\}$ (i.e. $E_{0}$ is not empty) then $v\left(\mathcal{T}_{0}, \theta\right)=f$. Hence $\mathcal{W}$ is not a model of $s$. We end the proof by showing that $\mathcal{W}$ is a model of $\mathcal{S}$. So let $\psi_{1}, \ldots, \psi_{n} \Rightarrow E \in \mathcal{S}$ and let $\mathcal{T} \in W$, where $\mathcal{T}$ is $F$-maximal. Assume by way of contradiction that $\psi_{1}, \ldots, \psi_{n} \Rightarrow E$ is not locally true in $\mathcal{T}$. Therefore, $v\left(\mathcal{T}, \psi_{i}\right)=t$ for $1 \leq i \leq n$. By (c), for every $1 \leq i \leq n$ there is a finite $\Gamma_{i} \subseteq \mathcal{T}$ such that $\Gamma_{i} \Rightarrow \psi_{i}$ has an $\mathcal{S}$-proof from $\mathcal{S}$. Now, there are two cases:

(1) Assume $E=\{\theta\}$. Since $\psi_{1}, \ldots, \psi_{n} \Rightarrow \theta$ is not locally true in $\mathcal{T}, v(\mathcal{T}, \theta)=f$. This implies (by (a)) that $\theta \notin \mathcal{T}$. Since $\mathcal{T}$ is $F$-maximal, it follows that there is a finite $\Delta \subseteq \mathcal{T}$ such that $\Delta, \theta \Rightarrow F$ has an $\mathcal{S}$-proof from $\mathcal{S}$. Now from $\Gamma_{i} \Rightarrow \psi_{i}(1 \leq i \leq n)$, $\Delta, \theta \Rightarrow F$, and $\psi_{1}, \ldots, \psi_{n} \Rightarrow \theta$ one can infer $\Gamma_{1}, \ldots, \Gamma_{n}, \Delta \Rightarrow F$ by $n+1 \mathcal{S}$-cuts (on $\psi_{1}, \ldots, \psi_{n}$ and $\left.\theta\right)$. Hence, $\Gamma_{1}, \ldots, \Gamma_{n}, \Delta \Rightarrow F$ has an $\mathcal{S}$-proof from $\mathcal{S}$.

(2) Assume $E$ is empty. $\Gamma_{1}, \ldots, \Gamma_{n} \Rightarrow$ follows from the sequents $\Gamma_{i} \Rightarrow \psi_{i}(1 \leq i \leq n)$ and $\psi_{1}, \ldots, \psi_{n} \Rightarrow$ by $n \mathcal{S}$-cuts (on $\psi_{1}, \ldots, \psi_{n}$ ). Using weakening (if $F$ is not empty), it follows that $\Gamma_{1}, \ldots, \Gamma_{n} \Rightarrow F$ has an $\mathcal{S}$-proof from $\mathcal{S}$.

In both cases we showed an $\mathcal{S}$-proof from $\mathcal{S}$ of a sequent of the form $\Gamma \Rightarrow F$, where $\Gamma \subseteq \mathcal{T}$. This contradicts the $F$-maximality of $\mathcal{T}$.

Remark 6.4. This proof suggests that weakening on the right side of sequents can be limited to apply only to negative sequents of the set of assumptions of the derivation. Recall that by proposition [3.9, $\mathcal{T} \vdash_{\mathbf{G}} E$ iff $\{\Rightarrow \psi \mid \psi \in \mathcal{T}\} \vdash_{\mathbf{G}}^{\text {seq }} \Rightarrow E$. Thus if one is only interested in consequence relations between formulas, there are no negative sequents in the set of assumptions, and so the right weakening rule is superfluous.

Theorem 6.5 (Soundness and Completeness). Every coherent canonical system $\mathbf{G}$ is strongly sound and complete with respect to the semantics of $\mathbf{G}$-legal frames. In other words: 
(1) $\mathcal{S} \vdash_{\mathbf{G}}^{\text {seq }} s$ iff $\mathcal{S} \vDash_{\mathbf{G}}^{\text {seq }} s$.

(2) $\mathcal{T} \vdash_{\mathbf{G}} E$ iff $\mathcal{T} \vDash_{\mathbf{G}} E$.

Proof. (1) is immediate from Theorem 6.3 and Theorem 6.1. (2) follows from (1) using the reductions given in Proposition 3.9 and Proposition 5.12 .

Corollary 6.6 (Compactness). Let $\mathbf{G}$ be a coherent canonical system. If $\mathcal{S} \vDash_{\mathbf{G}}^{\text {seq }} s$ then there exists a finite $\mathcal{S}^{\prime} \subseteq \mathcal{S}$ such that $\mathcal{S}^{\prime} \vDash_{\mathrm{G}}^{\text {seq }}$ s.

We use Theorem 6.3 to prove a general cut-elimination theorem.

Definition 6.7. Let $s$ be a sequent, $\mathcal{S}$ be a set of sequents, and $\mathbf{G}$ be a canonical system.

(1) $\mathbf{G}$ admits cut-elimination iff whenever $\vdash_{\mathbf{G}}^{\text {seq }} s$, there exists a proof of $s$ without cuts (i.e. there exists a $\emptyset$-proof).

(2) ([2]) $\mathbf{G}$ admits strong cut-elimination iff whenever $\mathcal{S} \vdash_{\mathbf{G}}^{\text {seq }} s$, there exists an $\mathcal{S}$-proof of $s$ from $\mathcal{S}$.

Notice that cut-elimination is a special case of strong cut-elimination with an empty $\mathcal{S}$. Also notice that by cut-elimination we mean here just the existence of proofs without (certain forms of) cuts, rather than an algorithm to transform a given proof to a cut-free one (for the assumption-free case the term cut-admissibility is sometimes used).

Theorem 6.8 (General Strong Cut-Elimination Theorem). Every coherent canonical system $\mathbf{G}$ admits strong cut-elimination.

Proof. Assume $\mathcal{S} \vdash_{\mathbf{G}}^{\text {seq }} s$. By Theorem 6.5, $\mathcal{S} \vDash_{\mathbf{G}}^{\text {seq }} s$, and so there does not exist a G-legal $\mathcal{L}$-frame which is model of $\mathcal{S}$, but not a model of $s$. By Theorem 6.3, there is an $\mathcal{S}$-proof of $s$ from $\mathcal{S}$.

Remark 6.9. In [2, a strengthening of the cut-elimination theorem was suggested for Gentzen's original systems for classical logic. The notion of a hyper-resolution rule (or hyper-cut rule) was defined, and it was proven that this special kind of cuts is the only one needed in derivations of a sequent from a non-empty set of sequents. Following the proof of Theorem 6.3, we can show the same in the present case. Let hyper-cut $t_{1}$ and hyper-cut ${ }_{2}$ be the rules which allow the following two derivations:

$$
\begin{gathered}
\psi_{1}, \ldots, \psi_{n} \Rightarrow \theta \quad \Gamma_{1} \Rightarrow \psi_{1} \quad \ldots \quad \Gamma_{n} \Rightarrow \psi_{n} \quad \Delta, \theta \Rightarrow F \\
\frac{\Gamma_{1}, \ldots, \Gamma_{n}, \Delta \Rightarrow F}{\psi_{1}, \ldots, \psi_{n} \Rightarrow \quad \Gamma_{1} \Rightarrow \psi_{1} \quad \ldots \quad \Gamma_{n} \Rightarrow \psi_{n}} \\
\Gamma_{1}, \ldots, \Gamma_{n} \Rightarrow
\end{gathered}
$$

Call $\psi_{1}, \ldots, \psi_{n} \Rightarrow E$, where $E=\{\theta\}$ in the first derivation and empty in the second, the nucleus of the rule. The last theorem can be strengthened as follows: if $\mathcal{S} \vdash_{\mathbf{G}}^{\text {seq }} s$, then there exists a proof of $s$ from $\mathcal{S}$, which uses only axioms, canonical rules, weakenings and hyper-cuts with elements of $\mathcal{S}$ as nuclei.

Corollary 6.10. The conditions below are equivalent for a canonical system $\mathbf{G}$ :

(1) $\mathbf{G}$ is strongly consistent.

(2) $\mathbf{G}$ is coherent.

(3) $\mathbf{G}$ admits strong cut-elimination. 
Proof. (1) implies (2) by Theorem 4.10, (2) implies (3) by Theorem 6.8. Finally, in a canonical system the only sequents which are provable from $\left\{\left(\Rightarrow p_{1}\right),\left(p_{2} \Rightarrow\right)\right\}$ using only cuts on $p_{1}$ or $p_{2}$ are: axioms, sequents of the form $\Gamma \Rightarrow p_{1}$, sequents of the form $\Gamma, p_{2} \Rightarrow E$, and sequents that contain a non-atomic formula. Thus there is no way to derive $\Rightarrow$ from $\left\{\left(\Rightarrow p_{1}\right),\left(p_{2} \Rightarrow\right)\right\}$, using only cuts on $p_{1}$ or $p_{2}$. Hence $(3)$ implies (1).

Corollary 6.11. If $\mathbf{G}$ is a coherent canonical system in $\mathcal{L}$ then $\left\langle\mathcal{L}, \vDash_{\mathbf{G}}\right\rangle$ (or equivalently $\left.\left\langle\mathcal{L}, \vdash_{\mathbf{G}}\right\rangle\right)$ is an extended logic.

6.1. Strict Canonical Systems. In [4] strict single-conclusion canonical systems were investigated. These systems are canonical systems, in which derivations can only contain definite sequents. Now we show that the results of [4] about these systems can be derived from results of the present paper. For this purpose, we concentrate on a smaller set of canonical systems, for which we are able to strengthen Corollary 6.10.

Definition 6.12. A canonical system is called definite if its right-introduction rules have only definite clauses as premises, and its left-introduction rules have only definite clauses as hard premises.

Example 6.13. Every canonical system in which the set of logical rules is a subset of the set of rules for $\supset, \perp, \leadsto, \triangleright$ (of Example 3.4) is definite.

Corollary 6.14. The conditions below are equivalent for a definite canonical system $\mathbf{G}$ :

(1) $\mathbf{G}$ is strongly consistent.

(2) $\mathbf{G}$ is coherent.

(3) $\mathbf{G}$ admits strong cut-elimination.

(4) $\mathbf{G}$ admits cut-elimination.

(5) $\mathbf{G}$ is consistent.

Proof. (1),(2),(3) are equivalent by Corollary 6.10 for every canonical system. (3) trivially implies (4). (4) implies (5), since in a canonical system there is no way to derive $p_{1} \Rightarrow p_{2}$ without using cuts. Finally, a proof similar to that of Theorem 1 in [4], (or Theorem 4.10 of this paper) shows that (5) implies (2).

Remark 6.15. Strong cut-elimination and cut-elimination are not equivalent in the general case. To see this, consider the system $\mathbf{G}$ given in Example 4.7. As explained there, a sequent $\Gamma \Rightarrow E$ can be proved in $\mathbf{G}$ from no assumptions iff it is of the form $\Gamma \Rightarrow \psi$, where $\circ^{n} \psi \in \Gamma$ for some $n \geq 0$. It is easy to see that every sequent of this form can be proved without using cuts, and so $\mathbf{G}$ admits cut-elimination. However, $\mathbf{G}$ does not admit strong cut-elimination. For example, one must apply cut on op to derive the empty sequent from the sequent $p_{1} \Rightarrow$.

To derive results about strict canonical systems, we prove the following lemma.

Lemma 6.16. Let $\mathbf{G}$ be a definite canonical system, and let $\mathcal{S} \cup\{s\}$ be a set of definite sequents. If there exists a proof $P$ of $s$ from $\mathcal{S}$ in $\mathbf{G}$, then there also exists a proof $P^{\prime}$ of $s$ from $\mathcal{S}$ in which every sequent is a definite sequent, and every cut formula in $P^{\prime}$ also serves as a cut-formula in $P$. 
Proof. It is easy to see that starting from definite assumptions, the only way one can produce a negative sequent in a definite canonical system is by an application of a left-introduction rule of the form:

$$
\frac{\left\{\Gamma, \sigma\left(\Pi_{i}\right) \Rightarrow \sigma\left(E_{i}\right)\right\}_{1 \leq i \leq m} \quad\left\{\Gamma, \sigma\left(\Sigma_{i}\right) \Rightarrow\right\}_{1 \leq i \leq k}}{\Gamma, \sigma\left(\diamond\left(p_{1}, \ldots, p_{n}\right)\right) \Rightarrow}
$$

Since $\mathbf{G}$ is definite, the sequent inferred in steps of this kind cannot be used in the rest of the proof, unless right weakening is applied on a descendant of this sequent. Applying the same weakening before steps of this kind will turn the sequent into a definite one, keeping the rest of the proof valid. Finally, this modification does not affect the set of cut-formulas used in the proof.

Now define a new strict provability relation $\vdash_{\mathbf{G}}^{s e q_{1}}$ for definite canonical systems. $\vdash_{\mathbf{G}}^{s e q_{1}}$ is defined as in Definition 3.6, except that it allows only definite sequents in proofs. By Lemma 6.16 it immediately follows that a definite system admits cut-elimination with respect to $\vdash_{\mathbf{G}}^{s e q_{1}}$, iff it admits cut-elimination with respect to $\vdash_{\mathbf{G}}^{s e q}$. The same applies to strong cutelimination and consistency. Therefore for definite canonical systems, Corollary 6.14] ensures that coherence, cut-elimination, strong cut-elimination, and consistency 5 are equivalent also with respect to $\vdash_{\mathbf{G}}^{s e q_{1}}$.

\section{Analycity and Decidability}

In this section we show that the semantics of $\mathbf{G}$-legal frames is analytic in the intuitive sense described in Remark 5.3 .

Theorem 7.1 (Analycity). Let $\mathcal{U}_{1}, \mathcal{U}_{2}$ be sets of formulas closed under subformulas, such that $\mathcal{U}_{1} \subset \mathcal{U}_{2}$. Let $\mathbf{G}$ be a coherent canonical system for $\mathcal{L}$. The semantics of $\mathbf{G}$-legal frames is analytic in the following sense: If $\mathcal{W}_{1}=\left\langle W, \leq, v_{1}\right\rangle$ is a $\mathbf{G}$-legal $\mathcal{U}_{1}$-semiframe, then $v_{1}$ can be extended to a function $v_{2}$ so that $\mathcal{W}_{2}=\left\langle W, \leq, v_{2}\right\rangle$ is a $\mathbf{G}$-legal $\mathcal{U}_{2}$-semiframe.

Proof. Similar to the proof of Theorem 6 from [4].

Remark 7.2. In particular, the last theorem shows that every $\mathbf{G}$-legal $\mathcal{U}$-semiframe, can be extended to a $\mathbf{G}$-legal $\mathcal{L}$-frame.

The following two theorems are consequences of Theorem 7.1 and the soundness and completeness theorems.

Theorem 7.3 (Conservativity). Let $\mathbf{G}_{\mathbf{1}}$ be a coherent canonical system in a language $\mathcal{L}_{1}$, and let $\mathbf{G}_{\mathbf{2}}$ be a coherent canonical system in a language $\mathcal{L}_{2}$. Assume that $\mathcal{L}_{2}$ is an extension of $\mathcal{L}_{1}$ by some set of connectives, and that $\mathbf{G}_{\mathbf{2}}$ is obtained from $\mathbf{G}_{\mathbf{1}}$ by adding to the latter canonical rules for connectives in $\mathcal{L}_{2}-\mathcal{L}_{1}$. Then $\mathbf{G}_{\mathbf{2}}$ is a conservative extension of $\mathbf{G}_{\mathbf{1}}$ (i.e.: if all sequents in $\mathcal{S} \cup s$ are in $\mathcal{L}_{1}$ then $\mathcal{S} \vdash_{\mathbf{G}_{1}}^{\text {seq }}$ s iff $\mathcal{S} \vdash_{\mathbf{G}_{\mathbf{2}}}^{\text {seq }}$ s).

Proof. Suppose that $\mathcal{S} \nvdash_{\mathbf{G}_{1}}^{\text {seq }} s$. Then there is $\mathbf{G}_{\mathbf{1}_{1}}$-legal model $\mathcal{W}$ of $\mathcal{S}$ which is not a model of $s$. Since the set of formulas of $\mathcal{L}_{1}$ is a subset of the set of formulas of $\mathcal{L}_{2}$ which is closed under subformulas, Theorem 7.1 implies that $\mathcal{W}$ can be extended to a $\mathbf{G}_{\mathbf{2}}$-legal model of $\mathcal{S}$ which is not a model of $s$. Hence $\mathcal{S} \nvdash_{\mathbf{G}_{2}}^{s e q} s$.

\footnotetext{
${ }^{5}$ Note that strong consistency is trivial in this case, since the empty sequent is not allowed to appear in derivations.
} 
Theorem 7.4 (Decidability). Let $\mathbf{G}$ be a coherent canonical system. Then $\mathbf{G}$ is strongly decidable: Given a finite set $\mathcal{S}$ of sequents, and a sequent $s$, it is decidable whether $\mathcal{S} \vdash_{\mathbf{G}}^{\text {seq }} s$ or not.

Proof. Let $\mathcal{U}$ be the set of subformulas in $\mathcal{S} \cup\{s\}$. From Theorem 7.1 and the proof of Theorem 6.3 it easily follows that in order to decide whether $\mathcal{S} \vdash_{\mathbf{G}}^{\text {seq }} s$ it suffices to check all triples of the form $\left\langle W, \subseteq, v^{\prime}\right\rangle$ where $W \subseteq 2^{\mathcal{U}}$ and $v^{\prime}: W \times \mathcal{U} \rightarrow\{t, f\}$, and see if any of them is a $\mathbf{G}$-legal $\mathcal{U}$-semiframe which is a model of $\mathcal{S}$ but not a model of $s$.

Remark 7.5. The last two theorems can also be proved directly from the cut-elimination theorem.

Strong conservativity and strong decidability of $\vdash_{\mathbf{G}}$ and $\vDash_{\mathbf{G}}$ are easy corollaries of the previous theorems and the reductions given in Proposition 3.9 and Proposition 5.12 .

\section{Conclusions and Further Work}

Now we present our answer to the question from the introduction: "what is a basic constructive connective?".

\section{A basic constructive connective is a connective defined by a set of rules in some coherent canonical system.}

Theorem 6.8 ensures that the proof-theoretic criterion for constructivity, described in the introduction, is met. Theorem 7.3 ensures that a set of rules for some connective can indeed be seen as a definition of that connective, because it shows that in coherent canonical systems the same set of rules defines the same connective regardless of the rules for the other connectives.

In Section 5, the proof-theoretic characterization of basic constructive connectives was matched by a (non-deterministic) Kripke-style semantics. This semantics is modular, allowing to separate the semantic effect of each derivation rule. However, we did not provide there an independent semantic characterization of (basic) constructive connectives. We leave this issue to a future work. Another future goal is to extend our results to first-order logic, and identify constructive quantifiers as well (for semi-classical quantifiers this was done in [5]).

\section{ACKNOWLEDGEMENTS}

We are grateful to two anonymous referees for their helpful suggestions and comments. This research was supported by The Israel Science Foundation (grant no. 280-10).

\section{REFERENCES}

[1] Avron, A.: Simple Consequence Relations. Information and Computation 92, 105-139 (1991).

[2] Avron, A.: Gentzen-Type Systems, Resolution and Tableaux. Journal of Automated Reasoning 10, 265-281 (1993).

[3] Avron, A., Lev, I.: Non-deterministic Multiple-valued Structures. Journal of Logic and Computation 15, 24-261 (2005). A partial conference version in Goré, R., Leitsch, A., Nipkow, T., (eds.): Proceedings of IJCAR 2001. LNCS (LNAI), vol. 2083, pp. 529-544. Springer, Heidelberg (2001). 
[4] Avron, A., Lahav, O.: Strict Canonical constructive systems. In Blass, A., Dershowitz, N., Reisig, W. (eds.): Fields of Logic and Computation: Essays Dedicated to Yuri Gurevich on the Occasion of His 70th Birthday, 75-94, Lecture Notes in Computer Science, volume 6300, Springer-Verlag, 2010. A conference version in M. Giese, A. Waaler (eds.): Proceedings of TABLEAUX 2009, 62-76, LNAI 5607, Springer (2009).

[5] Avron, A., Zamansky, A.: Canonical Gentzen-type calculi with (n,k)-ary quantifiers. Logical Methods in Computer Science 4, 1-23 (2008).

[6] Belnap, N. D.: Tonk, Plonk and Plink. Analysis 22, 130-134 (1962).

[7] Bowen, K. A.: An extension of the intuitionistic propositional calculus. Indagationes Mathematicae 33, 287-294 (1971).

[8] Ciabattoni, A., Terui, K.: Towards a Semantic Characterization of Cut-Elimination. Studia Logica 82, 95-119 (2006).

[9] Gentzen, G.: Investigations into Logical Deduction. In: Szabo, M.E. (ed.) The Collected Works of Gerhard Gentzen, pp. 68-131. North Holland, Amsterdam (1969).

[10] Gurevich, Y., Neeman, I.: The Infon Logic: the Propositional Case. To appear in ACM Transactions on Computation Logic 12 (2011). An earlier version in Bulletin of European Association of Theoretical Computer Science, number 98 (2009) 150-178.

[11] Kripke, S.: Semantical Analysis of Intuitionistic Logic I. In: Crossly, J., Dummett, M. (eds.) Formal Systems and Recursive Functions, pp. 92-129. North-Holland, Amsterdam (1965).

[12] McCullough, D.P.: Logical connectives for intuitionistic propositional logic. Journal of Symbolic Logic 36(1), 15-20 (1971).

[13] Prior, A.N.: The Runabout Inference Ticket. Analysis 21, 38-39 (1960).

[14] Sundholm, G.: Proof theory and Meaning. In: Gabbay, D.M., Guenthner, F. (eds.) Handbook of Philosophical Logic, vol. 9, pp. 165-198 (2002). 